OPEN ACCESS

Edited by:

Keqiang Wu,

National Taiwan University, Taiwan

Reviewed by:

Fan Chen,

Institute of Genetics and Developmental Biology (CAS), China Yuxin $\mathrm{Hu}$,

Institute of Botany (CAS), China

${ }^{*}$ Correspondence: Huiru Peng

penghuiru@cau.edu.cn

${ }^{\dagger}$ These authors have contributed equally to this work.

Specialty section: This article was submitted to Plant Genetics and Genomics,

a section of the journal

Frontiers in Plant Science

Received: 25 November 2016 Accepted: 27 January 2017

Published: 08 February 2017

Citation:

Zhao Y, Tian X, Li Y, Zhang L,

Guan $P$, Kou $X$, Wang $X$, Xin $M$,

Hu Z, Yao Y, Ni Z, Sun $Q$ and

Peng $H$ (2017) Molecular and Functional Characterization of Wheat ARGOS Genes Influencing Plant Growth and Stress Tolerance.

Front. Plant Sci. 8:170. doi: 10.3389/fpls.2017.00170

\section{Molecular and Functional Characterization of Wheat ARGOS Genes Influencing Plant Growth and Stress Tolerance}

\author{
Yue Zhao', Xuejun Tian', Yuanyuan Li, Liyuan Zhang, Panfeng Guan, Xiaoxia Kou, \\ Xiaobo Wang, Mingming Xin, Zhaorong Hu, Yingyin Yao, Zhongfu Ni, Qixin Sun and \\ Huiru Peng*
}

State Key Laboratory for Agrobiotechnology and Key Laboratory of Crop Heterosis and Utilization, Beijing Key Laboratory of Crop Genetic Improvement, Department of Plant Genetics and Breeding, China Agricultural University, Beijing, China

Auxin Regulated Gene involved in Organ Size (ARGOS) is significantly and positively associated with organ size and is involved in abiotic stress responses in plants. However, no studies on wheat $A R G O S$ genes have been reported to date. In the present study, three TaARGOS homoeologous genes were isolated and located on chromosomes 4A, $4 B$, and $4 D$ of bread wheat, all of which are highly conserved in wheat and its wild relatives. Comparisons of gene expression in different tissues demonstrated that the TaARGOSs were mainly expressed in the stem. Furthermore, the TaARGOS transcripts were significantly induced by drought, salinity, and various phytohormones. Transient expression of the TaARGOS-D protein in wheat protoplasts showed that TaARGOS$D$ localized to the endoplasmic reticulum. Moreover, overexpression of TaARGOS-D in Arabidopsis resulted in an enhanced germination rate, larger rosette diameter, increased rosette leaf area, and higher silique number than in wild-type (WT) plants. The roles of TaARGOS-D in the control of plant growth were further studied via RNA-seq, and it was found that 105 genes were differentially expressed; most of these genes were involved in 'developmental processes.' Interestingly, we also found that overexpression of TaARGOS-D in Arabidopsis improved drought and salinity tolerance and insensitivity to ABA relative to that in WT plants. Taken together, these results demonstrate that the TaARGOSs are involved in seed germination, seedling growth, and abiotic stress tolerance.

\section{Keywords: TaARGOS, transgenic gene, abiotic stress, ABA, wheat, Arabidopsis}

\section{INTRODUCTION}

An explosion in the global demand and consumption of agricultural crops for food, feed, and fuel, originating from the rapid increases in the global population and emerging economies, has made increased crop productivity a primary goal of breeding programs (Rosegrant and Cline, 2003; Edgerton, 2009). Crop yield improvements are either the result of increased total biomass 
production (larger plants tend to produce greater yields), an increased harvest index, or both (Quarrie et al., 2006; Fabre et al., 2016). Therefore, genes that control organ size and lead to higher yield are important targets of crop breeding (Gonzalez et al., 2009).

The ORGAN SIZE RELATED (OSR) family is known to promote plant organ growth and regulate the expression of organ size genes (Hu et al., 2003, 2006; Feng et al., 2011; Qin et al., 2014). Furthermore, previous data have shown that the OSR family is involved in the response to abiotic stresses (Guo et al., 2014; Rai et al., 2015; Shi et al., 2015, 2016a,b). In Arabidopsis, AUXIN REGULATED GENE INVOLVED IN ORGAN SIZE (ARGOS), the founding member of the OSR homologs, was identified as an auxin-induced gene that is expressed in developing organs. AtARGOS encodes a predicted integral membrane protein, and its overexpression is sufficient to increase organ size by stimulating cell proliferation; conversely, down-regulation causes reduced organ growth. AtARGOS promotes plant organ growth mainly by enhancing the continuous expression of $A N T$ and CycD3;1 (Hu et al., 2003). An earlier study indicated that the AtARGOS homolog ARGOS-LIKE (AtARL) also regulates lateral organ size. Increased $A t A R L$ expression is sufficient to cause organ enlargement due to an increase in cell size, while reduced AtARL expression leads to smaller organs with less expanded cells. In contrast to AtARGOS mediation of auxin effects on growth, AtARL was hypothesized to function downstream of brassinosteroids (BR; Hu et al., 2006). There are four OSR homologs in Arabidopsis, and other members of this family (OSR1 and OSR2) are also known to promote plant organ growth when overexpressed in Arabidopsis (Feng et al., 2011; Qin et al., 2014). In rice, five OSR members were identified including OsARGOS (Feng et al., 2011). It was found that overexpression of OsARGOS in Arabidopsis increases organ growth as well (Wang et al., 2009). The $A R G O S$ gene exists as eight copies in maize. Overexpression of ZmARGOS1 in maize enhances maize organ growth and increases yield (Guo et al., 2014). Furthermore, maize ARGOSs have been found to be related to abiotic stress (Guo et al., 2014; Rai et al., 2015; Shi et al., 2015, 2016a,b). For example, overexpression of ZmARGOS1 and ZmARGOS8 significantly improves tolerance to drought stress through an ethylenedependent regulation pathway in Arabidopsis and maize. Additionally, maize plants overexpressing ZmARGOS8 exhibit a greater grain yield than WT controls under both drought stress and well-watered conditions (Shi et al., 2015). Recently, Shi et al. (2016a) demonstrated that ZmARGOS1 and ZmARGOS8 modulate ethylene signal transduction through REVERSIONTO-ETHYLENE SENSITIVITY1-LIKE (RTL) proteins (Shi et al., 2016a). However, in wheat (Triticum aestivum L. $2 n=6 x=42$, genomes AABBDD), an important food crop around the world, no ARGOS genes have been reported.

In the current study, we report the identification, physical localization, and expression patterns of ARGOS homoeologous genes in bread wheat. Additionally, the subcellular localization of TaARGOS-D was determined, and the effects of overexpression of TaARGOS-D in Arabidopsis on plant growth and stress tolerance were studied.

\section{MATERIALS AND METHODS}

\section{Plant Materials and Stress Treatments}

Wheat cv. Jingdong6 was used for gene cloning and expression analyses. Seeds were sterilized in a $1 \% \mathrm{NaClO}$ solution and incubated for 1 day in sterile distilled water at $22^{\circ} \mathrm{C}$ in the dark. After germination, the seedlings were grown in a greenhouse $\left(22^{\circ} \mathrm{C}, 16 \mathrm{~h}\right.$ photoperiod). For drought, salt, and exogenous hormone treatments, 7-day-old seedlings were transferred to a water solution containing 20\% PEG6000, $200 \mathrm{mM} \mathrm{NaCl}$, $200 \mu \mathrm{M}$ ABA, $100 \mu \mathrm{M}$ methyl jasmonate (MeJA), $50 \mu \mathrm{M}$ $\alpha$-naphthaleneacetic acid (NAA), $50 \mu \mathrm{M}$ aminocyclopropane-1carboxylic acid (ACC, an ethylene precursor), $50 \mu \mathrm{M}$ gibberellic acid $\left(\mathrm{GA}_{3}\right)$, or $10 \mathrm{nM} \mathrm{BR}$. All treatments were performed under the same conditions for $0,1,2,4,6,12$, and $24 \mathrm{~h}$. After exposure to stress, the leaves were immediately frozen in liquid nitrogen prior to expression analysis. To study the tissue-specific expression of the TaARGOSs, dry seeds, seedlings at 1-6 days after germination (DAG), and roots, stems, leaves, and young spikes (YS) in the booting stage were obtained from Jingdong6 wheat. Nine diploid progenitor species [three A genome T. urartu species, three S (possibly modified B) genome Aegilops speltoides species, and three D genome $A$. tauschii species], three tetraploid T. dicoccum species, and six common wheat cultivars were used for the sequence comparison analysis (Supplementary Table S1). Chinese Spring (CS) nulli-tetrasomic (NT) lines were employed to determine the chromosomal locations of each gene.

The Arabidopsis Columbia-0 ecotype was used as the WT. Seeds were surface sterilized with a $5 \% \mathrm{NaClO}$ solution and cold treated at $4^{\circ} \mathrm{C}$ for 3 days in the dark, then plated on Murashige and Skoog (MS) medium containing 3\% (w/v) sucrose and $0.8 \%$ agar. Seven-day-old seedlings were transferred to a growth chamber under $16 / 8 \mathrm{~h}$ light/dark conditions at $22^{\circ} \mathrm{C}$.

\section{Cloning of the TaARGOSs and Sequence Analysis}

The sequence of the Arabidopsis ARGOS gene was used as probe for BLAST searches against the wheat genome sequence database of the International Wheat Genome Sequencing Consortium (IWGSC) ${ }^{1}$ and the UniProt databases ${ }^{2}$. Based on the nucleotide sequence polymorphisms of the TaARGOSs, the genomespecific primer sets TaARGOS-AF1/TaARGOS-AR1, TaARGOSBF1/TaARGOS-BR1, and TaARGOS-DF1/TaARGOS-DR1 were used to obtain the full-length DNA sequences (including the $5^{\prime}$ and $3^{\prime}$ flanking regions) from chromosomes $4 \mathrm{~A}, 4 \mathrm{~B}$, and $4 \mathrm{D}$, respectively. Each primer set was tested with CS NT lines to determine the chromosomal locations of the amplified genes. All PCR primers used in this study are described in Supplementary Table S2.

Genomic DNA was isolated from young leaves using the CTAB method. The PCR assays were performed using highfidelity Primestar polymerase (TaKaRa, Dalian, China) under the following conditions: $98^{\circ} \mathrm{C}$ for $3 \mathrm{~min}$, followed by 35 cycles of

\footnotetext{
${ }^{1}$ http://www.wheatgenome.org/

${ }^{2}$ http://www.uniprot.org/
} 
$98^{\circ} \mathrm{C}$ for $15 \mathrm{~s}, 58^{\circ} \mathrm{C}$ for $15 \mathrm{~s}$, and $72^{\circ} \mathrm{C}$ for $90 \mathrm{~s}$, with a final extension at $72^{\circ} \mathrm{C}$ for $3 \mathrm{~min}$. The PCR products were recovered, cloned into the pMD18-T vector (TaKaRa, Dalian, China) and sequenced. Total RNA was extracted using the TRIzol reagent according to the manufacturer's protocol (TianGen, Beijing, China) and treated with DNase I to remove the contaminating genomic DNA. Two micrograms of total RNA were reverse transcribed using AMV Reverse Transcriptase (TaKaRa, Dalian, China) following the manufacturer's instructions. The cDNA was diluted and used as a template for RT-PCR or quantitative RTPCR (qRT-PCR). According to the acquired genomic sequences, gene-specific primer sets were designed to amplify the TaARGOS cDNA sequences from wheat cv. Jingdong6. The PCR conditions and sequencing procedures were as described above.

The primers employed for PCR were designed using DNAMAN software. Alignment analysis was performed with ClustalW. Phylogenetic trees were constructed with MEGA6 software using the neighbour-joining (NJ) method (Tamura et al., 2013).

\section{Gene Expression Analysis}

Real-time qRT-PCR analysis was conducted using SYBR Premix $\mathrm{EX} \mathrm{Tag}^{\mathrm{TM}}$ (TaKaRa, Dalian, China) in a volume of $20 \mu \mathrm{l}$ in a Bio-Rad CFX96 real-time PCR detection system ${ }^{3}$. The PCR parameters were as follows: $94^{\circ} \mathrm{C}$ for $3 \mathrm{~min}$, followed by 40 cycles of $94^{\circ} \mathrm{C}$ for $15 \mathrm{~s}, 60^{\circ} \mathrm{C}$ for $20 \mathrm{~s}$, and $72^{\circ} \mathrm{C}$ for $20 \mathrm{~s}$. The wheat $\beta$-actin gene was used as a reference gene ( $\mathrm{Hu}$ et al., 2013). Each amplification reaction was repeated three times. Validation experiments were performed to demonstrate that the amplification efficiency of the TaARGOS-specific primers was approximately equal to the amplification efficiency of the endogenous reference primers. Quantification of the target gene expression was carried by the comparative CT method (Livak and Schmittgen, 2001). The primers employed for qRT-PCR are listed in Supplementary Table S2.

For the semi-qRT-PCR assay, the PCR conditions for the amplification of TaARGOS-D were as follows: $5 \mathrm{~min}$ at $94^{\circ} \mathrm{C}$, followed by 34 cycles of $20 \mathrm{~s}$ at $94^{\circ} \mathrm{C}, 20 \mathrm{~s}$ at $60^{\circ} \mathrm{C}$, and $20 \mathrm{~s}$ at $72^{\circ} \mathrm{C}$. The same conditions were used for the amplification of Actin2 of Arabidopsis, except that the number of PCR cycles was decreased to 25 .

\section{Subcellular Localization Analysis}

The full-length coding sequence of TaARGOS-D without the stop codon was obtained through PCR amplification with high-fidelity Primestar polymerase and primers with restriction enzyme sites (HindIII and XbaI). The double-digested PCR products were inserted into the $\mathrm{N}$-terminus of the green fluorescent protein (GFP) gene under the control of the cauliflower mosaic virus (CaMV) 35S promoter. The construct was confirmed by sequencing and transformed into wheat protoplasts via the PEGmediated transformation method (Yoo et al., 2007; Shan et al., 2014). The transformed wheat protoplasts were cultured at $25^{\circ} \mathrm{C}$ for $18 \mathrm{~h}$ and observed under a confocal microscope (Eclipse TE2000, Nikon).

\footnotetext{
${ }^{3}$ http://www.bio-rad.com/
}

\section{GUS Histochemical Staining}

The TaARGOS-D promoter-GUS reporter plasmid was constructed by cloning a PCR-amplified DNA fragment containing the TaARGOS-D 5' sequence (1670 bp) into the GUS reporter plasmid pCAMBIA1381Z. Histochemical GUS staining was performed as described previously (Jefferson et al., 1987). Whole transgenic Arabidopsis seedlings or different tissues were immersed in staining buffer at $37^{\circ} \mathrm{C}$ in the dark from $3 \mathrm{~h}$ to overnight depending on the experimental requirements. For stress treatment, the 7-day-old transgenic seedlings were transferred to one of the following MS plates: 10\% PEG6000, $150 \mathrm{mM} \mathrm{NaCl}, 100 \mu \mathrm{M}$ ABA, $10 \mu \mathrm{M}$ MeJA, $50 \mu \mathrm{M}$ NAA, $10 \mu \mathrm{M}$ ACC, or $50 \mu \mathrm{M}$ GA for $6 \mathrm{~h}$ before histochemical staining.

\section{Construction of Transgenic Plants}

The full-length coding region of TaARGOS-D cDNA was amplified through PCR using gene-specific primers containing attB sites (Supplementary Table S2, the attB sites are underlined). The PCR product was cloned into the Gateway plant expression vector pB2GW7 harboring the CaMV 35S promoter. The construct was then sequenced and introduced into Agrobacterium GV3101 and transformed into Arabidopsis plants via the floral dip method (Clough and Bent, 1998). The positive transgenic plants were screened and then further identified through RT-PCR.

\section{Microscopy Counts of Leaf Epidermal Cells}

The fifth leaf was collected from greenhouse-grown 3-weekold plants. Chlorophyll was removed by washing the leaf $2 \sim 3$ times until the washing solution (70\% ethanol) remained clear. The leaves were then soaked in Hoyer's solution to make the tissue transparent. Next, a $1-\mathrm{cm}^{2}$ section was removed from the midpoint of these fixed leaves, cleared in a saturated solution of chloral hydrate, and mounted on a glass slide under a cover glass. Images were collected using a Nikon Ti-U microscope. All whole cells captured in the field were counted.

\section{RNA-Seq Analysis}

RNA-seq analysis was carried out using an Arabidopsis transgenic line (L3). Total RNA was isolated from the 6-day-old seedlings of L3 and WT plants grown in Petri dishes under normal conditions using the TRIzol reagent (TianGen, Beijing, China). cDNA synthesis was performed according to the previously described method. Paired-end (PE) sequencing libraries were prepared using the TruSeq RNA Sample Preparation Kit v2 (Illumina, San Diego, CA, USA). Sequencing was performed on the Illumina HiSeq 2000 platform. Raw reads from the libraries were filtered, and the clean reads from each library were then mapped to the reference Arabidopsis genome (TAIR 10) using bowtie2 (Langmead and Salzberg, 2012). The reliability of the RNA-seq data was confirmed by calculating Pearson's correlation coefficient between each pair of biological replicates. Gene expression levels were measured based on RPKM (reads per kilobase of exon model per million mapped reads) values. Differentially expressed genes (DEGs) in the six libraries from 
wild-type and L3 plants (three libraries from three biological replicates for each sample) were identified according to false discovery rate (FDR) values $\leq 0.05$ and fold change values $\geq 2$. Pathway and Gene Ontology (GO) analyses were performed using the agriGO database $e^{4}$.

\section{Stress Treatment Analysis of Transgenic Arabidopsis Plants}

For the germination assays under stress treatment, approximately 64 seeds from each of the transgenic lines and WT plants were surface-sterilized and then planted on MS agar medium containing either $1 \mu \mathrm{M} \mathrm{ABA}, 10 \%$ PEG6000, or $100 \mathrm{mM} \mathrm{NaCl}$. The data were collected every day, and photographs were taken on the 7 th day. Germination was defined as a clear sign of the emergence of the radicle tip. The germination analyses were repeated three times.

For the salt and drought stress treatments, surface-sterilized transgenic and WT seeds were planted on MS medium plates and grown under standard culture conditions with white fluorescent light (16 h light $/ 8 \mathrm{~h}$ dark) at $22^{\circ} \mathrm{C}$. Seven-day-old seedlings were transferred to MS medium containing either 0 or $10 \%$ PEG6000 or $150 \mathrm{mM} \mathrm{NaCl}$ and then planted vertically for 5 days, after which the root lengths and fresh weights of the transgenic and WT plants were measured and photographed. Additionally, 7-day-old seedlings were transferred to mixed soil (rich soil: vermiculite $=2: 1, \mathrm{v} / \mathrm{v}$ ). Each pot contained four seedlings, which were grown for 2 weeks with sufficient watering. The plants were then subjected to drought stress treatment by withholding irrigation for 2 weeks. For salt stress analysis, the transgenic and WT plants were irrigated with a $200 \mathrm{mM} \mathrm{NaCl}$ solution once every 3 days for 2 weeks. The results were calculated based on three independent experiments.

\section{RESULTS}

\section{Isolation and Chromosomal Location of TaARGOS Homoeologous Genes}

Based on the sequence of the Arabidopsis ARGOS gene, three wheat contig sequences ( $T$. aestivum 4AL contig 346934, T. aestivum 4BS contig 186453, and T. aestivum 4DS contig 18687) were identified in the IWGSC databases. According to the observed BLAST hits, we designed genome-specific primer sets to obtain TaARGOS homoeologous genes from the A, B, and D genome sequences (Figure 1A). Each primer set was tested in CS NT lines. Amplification products were observed in four CS NT lines, with the exception of NT4A4B and NT4A4D, indicating that the TaARGOS amplified by the TaARGOS-AF1/TaARGOSAR1 primer set was located on chromosome 4A, and this gene was designated TaARGOS-A (KX768731). Likewise, TaARGOS$B$ (KX768732) and TaARGOS-D (KX768733), amplified by primer sets TaARGOS-BF1/TaARGOS-BR1 and TaARGOSDF1/TaARGOS-DR1, were located on chromosomes $4 \mathrm{~B}$ and 4D, respectively (Figure 1B). To obtain the cDNA sequences of these

\footnotetext{
${ }^{4}$ http://bioinfo.cau.edu.cn/agriGO/index.php
}

genes, we designed gene-specific primers to amplify the genes from the cDNA of wheat leaves. By comparing the genomic and cDNA sequences, we found that TaARGOS-A, TaARGOS- $B$, and TaARGOS-D consisted of one exon and contained a 333-bp open reading frame encoding 110 amino acid residues (Figure 2A). Multiple sequence alignments using DNAMAN revealed that the deduced polypeptides of the three TaARGOS homoeologous proteins shared $98.0 \%$ sequence similarity. In addition, we found that all three TaARGOS proteins contained an organ size-related (OSR) conserved domain in the C-terminal half of the protein, consistent with ARGOS proteins from various other species (Figure 2A).

We also searched the wheat OSR genes against the UniProt databases using the OSR domain sequence of the Arabidopsis ARGOS, and 12 uncharacterized wheat protein sequences were identified. Three out of the 12 identified sequences were the ones we cloned. Phylogenetic analysis of the deduced amino acid sequences with known OSR proteins in rice and Arabidopsis showed that the TaARGOSs were homologous to Arabidopsis AtARGOS, which were grouped into the same clade (Figure 2B). We next isolated and sequenced the genomic sequences of the TaARGOSs and their orthologs in diploid, tetraploid and hexaploid wheat. The TaARGOSs (including the coding and partial flanking regions) were amplified from 12 progenitor accessions and 6 modern hexaploid cultivars (Supplementary Table S1) using genome-specific primers. All sequences identified in hexaploid wheat were present in the corresponding genome donor species, and the coding regions exhibited no nucleotide differences in the A, B, and D genomes (Figure 3), which suggested that TaARGOSs are highly conserved during the polyploidization.

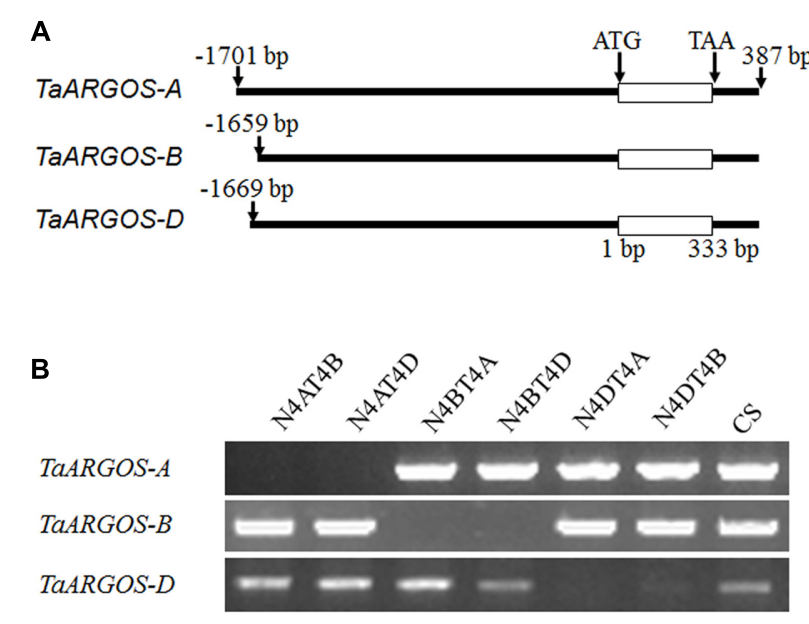

FIGURE 1 | Cloning regions of the TaARGOSs using genome-specific primers (A) and each primer set was tested on CS nulli-tetrasomic lines (B). Three sets of primers recognized as TaARGOS- $4 A,-4 B$, and $-4 D$ on chromosomes $4 \mathrm{~A}, 4 \mathrm{~B}$, and $4 \mathrm{D}$, respectively. N4AT4B: nullisomic 4A-tetrasomic 4B; N4AT4D: nullisomic 4A-tetrasomic 4D; N4BT4A: nullisomic 4B-tetrasomic 4A; N4BT4D: nullisomic 4B-tetrasomic 4D; N4DT4A: nullisomic 4D-tetrasomic 4A; N4DT4B: nullisomic 4D-tetrasomic 4B; CS:

Chinese Spring. 
A

TAARGOS-A
TAARGOS-B
TAARGOS-D
OSARGOS
ZMARGOS1
ZMARGOS8
AtARGOS
AtARL
AtOSR1
AtOSR2

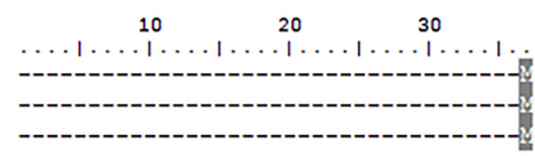

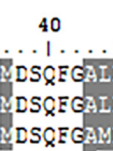

MSTTRP--------EDTCQLINS-------AAASPNRSAPSAAPSDMDEGSGTAASS-SRASTTSHSHCRATHRVVIEEEDD引PESSR 72
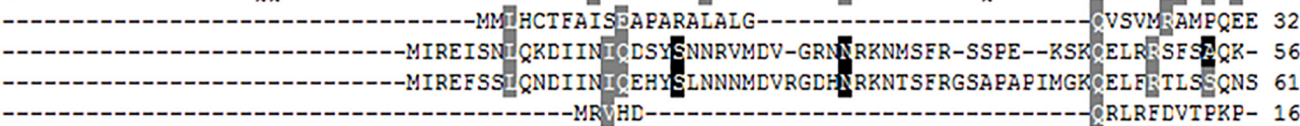

AtOSR2

OSR domain
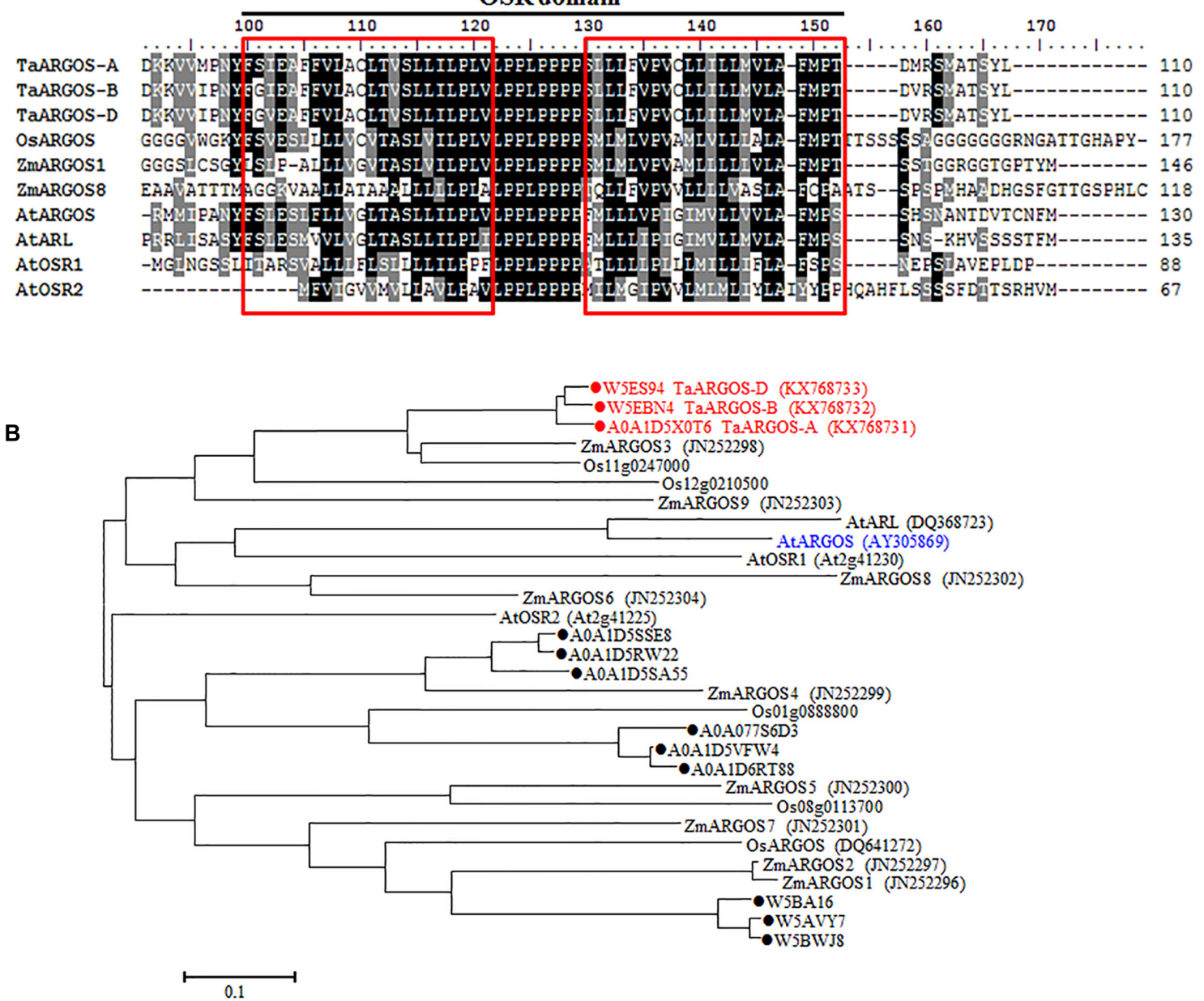

FIGURE 2 | Sequence alignment and phylogenetic analysis of the TaARGOSs and other ARGOS proteins. (A) Amino acid sequence alignment of the TaARGOSs and the ARGOS from Arabidopsis AtARGOS (Hu et al., 2003), AtARGOS-like (Hu et al., 2006), AtOSR1 (Feng et al., 2011), and AtOSR2 (Qin et al., 2014), rice OsARGOS (Wang et al., 2009), and maize ZmARGOS1 (Guo et al., 2014) and ZmARGOS8 (Shi et al., 2015). Consensus sequences (100 and $75 \%$ ) are exhibited in black and gray shading. The black line represents the conserved OSR domain. Red boxes indicate the putative transmembrane helices. (B) Phylogenetic tree based on amino acid sequences indicating the relationships of TaARGOSs with other plant ARGOS proteins. The accession numbers of the amino acid sequences are given in brackets. Twelve wheat protein sequences from the UniProt databases were indicated by dots. ZmARGOS2 is an allele variant of the ZmARGOS1 gene.

\section{Expression Analysis of TaARGOS Genes in Wheat}

To understand the temporal-spatial expression pattern of the TaARGOS homoeologous genes, we analyzed the expression patterns of the TaARGOSs in different wheat tissues. qRT-PCR analysis showed that the transcription levels of the TaARGOSs were different between tissues. The maximal expression levels of the TaARGOSs were detected in the stem at the booting stage, while moderate expression levels were detected in YS; very low expression levels were detected at early seed germination; and no expression was detected in dry seeds (Figure 4A). Interestingly, different expression patterns were observed for TaARGOS-A, TaARGOS-B, and TaARGOS-D, suggesting that these homeologs may play multiple roles at different developmental stages. 


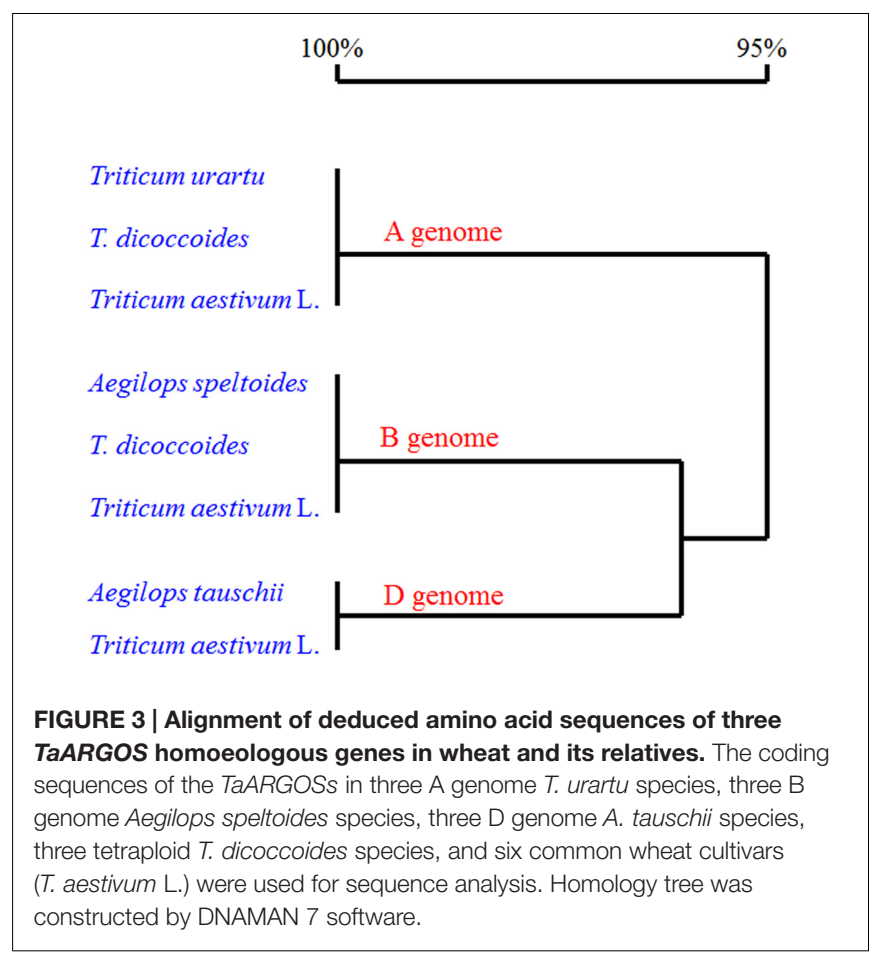

TaARGOS-D showed the highest expression level in all of the tested tissues (Figure 4A), indicating that it had a greater impact on regulating plant development than the other two homeologs. Thus, the TaARGOS-D gene was selected for further functional analysis.

Previous studies demonstrated that the Arabidopsis and maize ARGOS genes enhance plant drought tolerance and can be induced by various hormones (Qin et al., 2014; Shi et al., 2015). To determine whether the TaARGOSs function in stress responses in wheat, we assayed their expression following exposure of the plants to PEG, $\mathrm{NaCl}, \mathrm{ABA}, \mathrm{MeJA}, \mathrm{NAA}, \mathrm{ACC}$, $\mathrm{GA}_{3}$, and BR. As shown in Figure 4, the TaARGOSs were induced but were differentially expressed in response to abiotic stresses and hormones. Under PEG stress, the expression of the TaARGOSs peaked after $4 \mathrm{~h}$ (Figure 4B). Under salt stress, the expression of TaARGOS-A and TaARGOS-D increased and peaked at 4 and $6 \mathrm{~h}$, respectively, after which the level of mRNA accumulation gradually declined (Figure 4C). Following exogenous ABA treatment, the expression of the TaARGOSs was up-regulated at $4 \mathrm{~h}$ and peaked at $12 \mathrm{~h}$ (Figure 4D). Upon exogenous MeJA treatment, the expression of TaARGOS$A$ peaked rapidly at $6 \mathrm{~h}$ and then declined rapidly and reached pretreatment levels at $24 \mathrm{~h}$; however, TaARGOS$D$ expression was up-regulated at $4 \mathrm{~h}$ (Figure 4E). Under NAA treatment, the expression of the TaARGOSs increased gradually and peaked at $4 \mathrm{~h}$ (Figure 4F). The expression of the TaARGOSs was gradually up-regulated after exposure to exogenous ACC or $\mathrm{GA}_{3}$ and peaked at $6 \mathrm{~h}$ (Figures $4 \mathrm{G}, \mathbf{H}$ ). The expression of the TaARGOSs decreased after treatment with exogenous $B R$ and reached pretreatment levels after $4 \mathrm{~h}$ (Figure 4I).

\section{Promoter Isolation and Activity Analysis of TaARGOS-D}

To gain further insight into the mechanism responsible for the transcriptional regulation of the TaARGOSs, we isolated the sequence 1659-1701 bp upstream of the start codons of the three TaARGOSs using a PCR strategy. We searched for putative cis-acting elements in the promoter regions using PlantCARE ${ }^{5}$ (Figure 5A). A number of regulatory elements responding to defence and stress were recognized, including MBS and TC-rich binding sequences. In addition, an auxin-responsive element (AuxRR-core), a gibberellin-responsive element (P-box), an ethylene-responsive element (ERE), and an MeJA-responsive element (CGTCA-motif) were identified. The promoter region of TaARGOS-B was distinct from that of TaARGOS-A and TaARGOS-D. Compared with TaARGOS-A and TaARGOS-D, the auxin-responsive element was deleted at the same position in the promoter region of TaARGOS-B (Figure 5A).

To further study the expression pattern of the TaARGOSs, we prepared the pTaARGOS-D::GUS construct, with the GUS reporter gene driven by the TaARGOS-D promoter, and transferred it into Arabidopsis. GUS staining of transgenic Arabidopsis revealed that the TaARGOS-D promoter is very active in the leaf veins, hypocotyl, the trichomes of the first true leaves, roots and flowers (Figure 5B). Stress-responsive expression of TaARGOS- $D$ was further confirmed by histochemical staining in 7-day-old seedlings expressing the $p$ TaARGOS-D::GUS construct and treated with either $10 \%$ PEG6000, $150 \mathrm{mM} \mathrm{NaCl}, 100 \mu \mathrm{M}$ $\mathrm{ABA}, 10 \mu \mathrm{M}$ MeJA, $50 \mu \mathrm{M}$ NAA, $10 \mu \mathrm{M}$ ACC, or $50 \mu \mathrm{M} \mathrm{GA}_{3}$ for $6 \mathrm{~h}$ in MS agar medium. As shown in Figure 5C, enhanced GUS activity was observed in NaCl-, PEG- and exogenous hormonetreated seedlings compared with untreated controls.

\section{TaARGOS-D Localizes to the Endoplasmic Reticulum}

To determine the subcellular localization of the TaARGOS proteins in wheat cells, we transiently expressed the 35S::TaARGOS-D-GFP fusion protein in wheat protoplasts. As shown in Figure 6, the green fluorescence signal of the control 35S::GFP was uniformly distributed throughout the cell (upper panel), whereas TaARGOS-D-GFP exhibited a network structure (lower panel) consistent with the ER-localized GFP signals from the GFP: ARGOS and eGFP:MRN1 constructs (Go et al., 2012; Rai et al., 2015), indicating that TaARGOS-D localizes to the ER.

\section{Transgenic Overexpression of TaARGOS-D Increases Plant Growth}

To further understand the role of the TaARGOSs in plant growth, we generated transgenic Arabidopsis plants overexpressing TaARGOS-D under the control of the CaMV $35 S$ promoter. RTPCR was performed to assess the expression of TaARGOS-D in 11 T3 homozygous transgenic lines, and three independent lines with high expression levels (L3, L4, and L9) were used for further analysis (Supplementary Figure S1). In the germination process, the seed germination of the three transgenic lines was

\footnotetext{
${ }^{5}$ http://bioinformatics.psb.ugent.be/webtools/plantcare/html/
} 

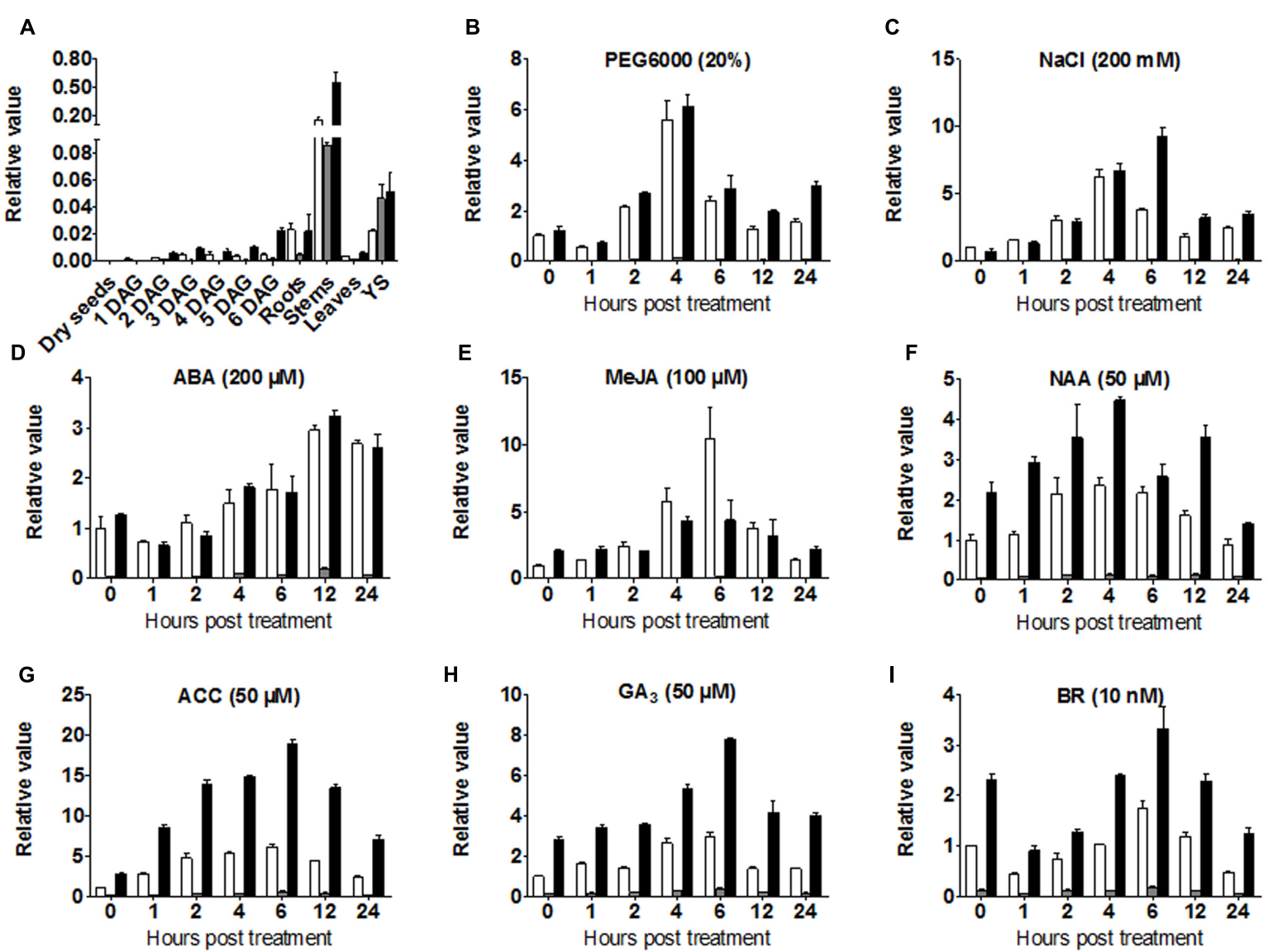

ए TaARGOS-A

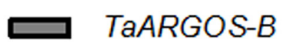

TaARGOS-D

FIGURE 4 | Expression pattern of the TaARGOS genes. (A) Expression pattern analysis by qRT-PCR of the three TaARGOS homoeologous genes in various wheat tissues. Total RNA was isolated from roots, stems, leaves, young spikes (YS), dry seeds, and seedlings at 1-6 days after germination (DAG). (B-I) qRT-PCR assessment of expression patterns of the wheat TaARGOS genes under PEG, NaCl, and exogenous ABA, MeJA, NAA, ACC, or GA 3 treatments. Total RNA was isolated from leaves of wheat seedlings. The $\beta$-actin gene was used as an internal reference. Error bars show standard deviation (SD) of three independent biological replicates.

significantly faster than that of the WT line (Supplementary Figure S2), with $90 \%$ of the seeds of the transgenic lines germinating in the $36 \mathrm{~h}$ after sowing, while only approximately $70 \%$ of the WT seeds germinated during the same time period. At the seedling stage, the transgenic plants initially grew larger than the WT plants, but the difference decreased gradually after the bolting stage, and the height of the transgenic plants was eventually only slightly greater than that of WT plants (Figures 7A,B). The fresh weight of the transgenic plants was markedly higher than that of the WT plants at the mature period (Figure 7C). A similar difference between transgenic lines and WT plants was observed in the number of siliques per plant (Figure 7D). Although silique size was indistinguishable, earlier bolting and flowering were observed in transgenic plants (Figure 7A). Thus, these data demonstrate that the observed enhancement of plant growth was due to a faster growth rate rather than an extended growth period.

Since the transgenic plant seedlings displayed large leaves, the adaxial side of the 5th leaves of 21-day-old WT and L3 plants was visualized using a Nikon Ti-U microscope, which was subsequently employed to measure the cell number in the leaves (Figures 7E,F). We counted the leaf epidermal cell number per unit area and the cell number within $1 \mathrm{~mm}^{2}$. The average number of cells in the transgenic lines was found to be the same as in the WT line, indicating that cell size was unchanged in the transgenic plants. The L3 leaves were approximately twice as big as the WT leaves. Therefore, we believe that the changes in overall plant size were due to a change in cell numbers rather than cell size. Similar mechanisms have been reported for Arabidopsis ARGOS gene (Hu et al., 2003).

\section{Transgenic Overexpression of TaARGOS-D Affects Development-Related Genes}

To investigate the possible molecular mechanisms underlying the effect of the TaARGOSs on plant growth, we used the RNAseq approach to identify genes showing altered expression levels 
A

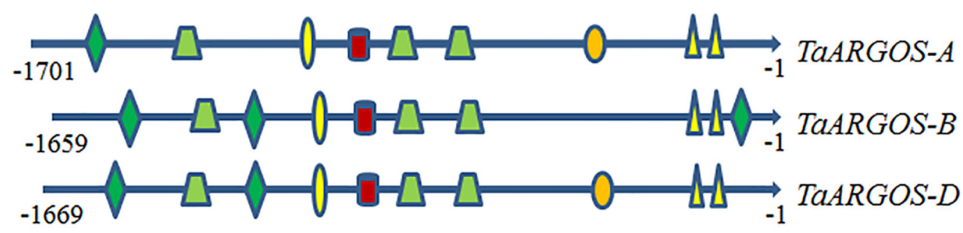

$\triangle$ MBS $\triangle$ TC-rich 0 p-box $\square$ MeJA $\quad$ ERE 0 AuxRR-core

B
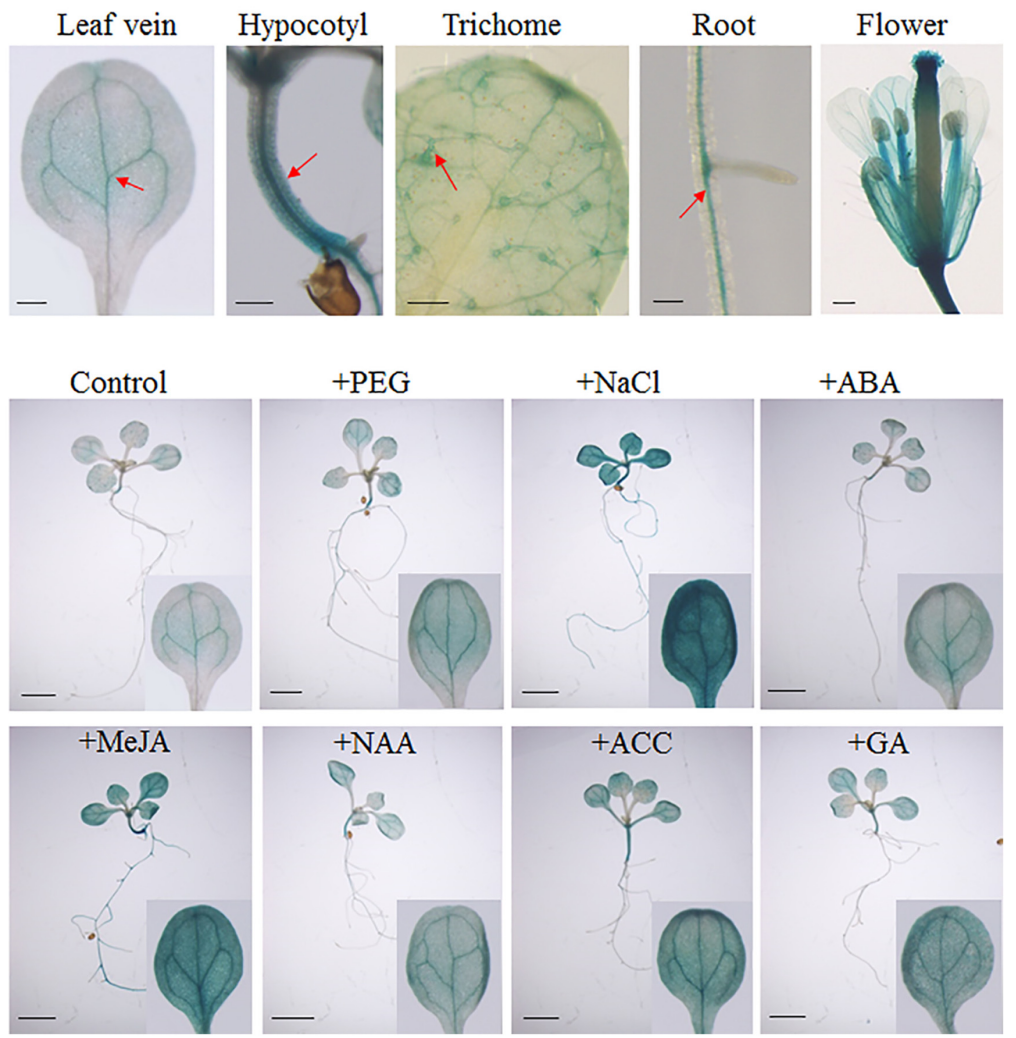

FIGURE 5 | Promoter isolation and activity analysis of the TaARGOSs (A) Cis-acting regulatory elements of promoter regions of TaARGOSs. (B) GUS staining of various pTaARGOS-D::GUS transgenic Arabidopsis tissues. GUS staining is mainly localized to leaf veins, hypocotyl, the trichomes of the first true leaves, roots, and flower (arrow). Scale bars $=0.2 \mathrm{~mm}$. (C) 7-day-old Arabidopsis pTaARGOS-D::GUS seedlings were grown on MS plates before transfer to either MS plates (Control), plates with 10\% PEG6000, 150mM NaCl, $100 \mu \mathrm{M} \mathrm{ABA,} 10 \mu \mathrm{M}$ MeJA, $50 \mu \mathrm{M}$ NAA, $10 \mu \mathrm{M}$ ACC, or $50 \mu \mathrm{M}$ GA3 and treated for 6 h. Enhanced GUS staining is visible in stresses treated seedlings. Scale bars $=2 \mathrm{~mm}$.

in the TaARGOS-D transgenic plants. Processing RNA samples using the Illumina HiSeq 2000 system yielded more than eight million reads, each $125 \mathrm{bp}$ in length, encompassing $2 \mathrm{~Gb}$ of sequence data for each sample, which were then mapped to the reference genome (Supplementary Figure S3A). The Pearson's correlation coefficients obtained by comparing three biological replicates of the either the WT or transgenic lines (L3), visualized in the correlation plot (Supplementary Figure S3B), are close to 1 . These analyses demonstrated that the raw RNA-seq data exhibited high levels of reliability and reproducibility.

The statistical analysis identified a total of 105 DEGs between the transgenic and WT plants using a $Q$-value $<0.05$ as a cutoff, among which 35 (33\%) were up-regulated, and 70 (67\%) were down-regulated, with fold changes higher than 2. Not surprisingly, growth and development genes dominated the list (Figure 8A, Supplementary Table S2). AtTIE1 encodes an EAR motif-containing protein that controls leaf development by affecting cell differentiation (Tao et al., 2013). In addition, we detected two operon-like gene clusters that were also upregulated in transgenic plants, which have been shown to be critical for growth and development in Arabidopsis (Field and Osbourn, 2008; Field et al., 2011; Go et al., 2012). The first operon-like gene cluster is essential for the synthesis of thalianol-derived triterpenes (the thalianol cluster), and consists 


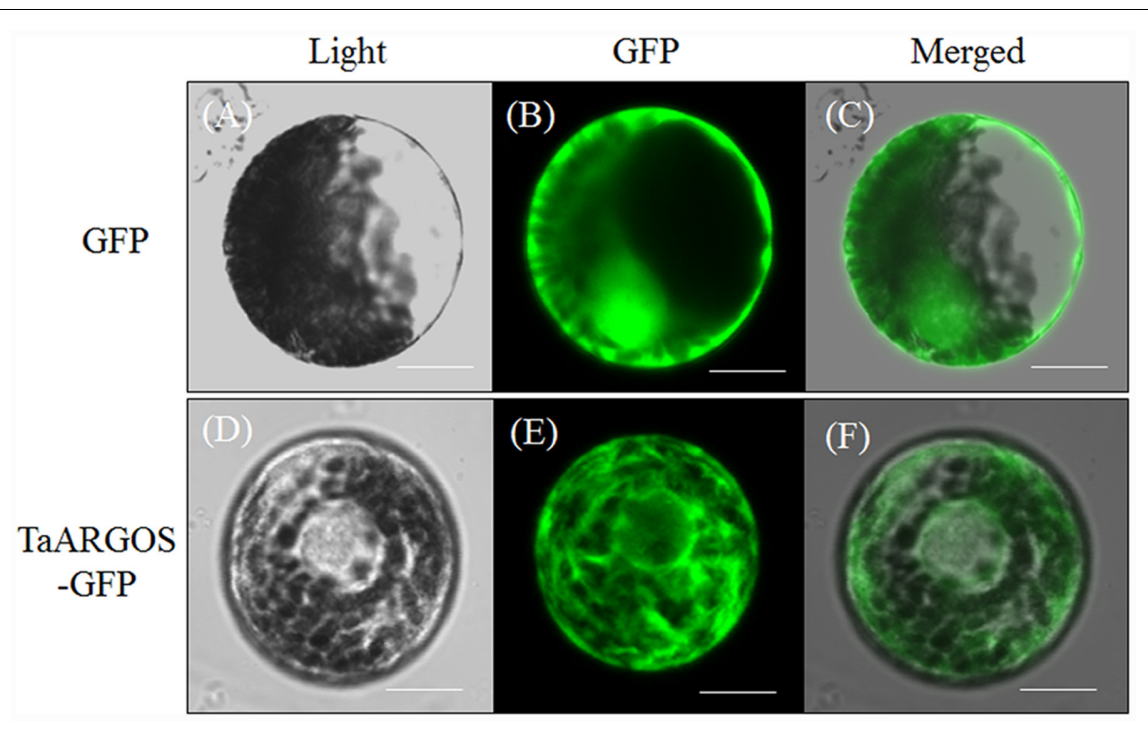

FIGURE 6 | Subcellular localization of the TaARGOS protein in wheat protoplasts. Photographs were taken in the light field (A and D), in the dark field for green fluorescence (B and $\mathbf{E}$ ), and in combination for morphology of the cells $(\mathbf{C}$ and $\mathbf{F})$. Bars $=10 \mu \mathrm{M}$.

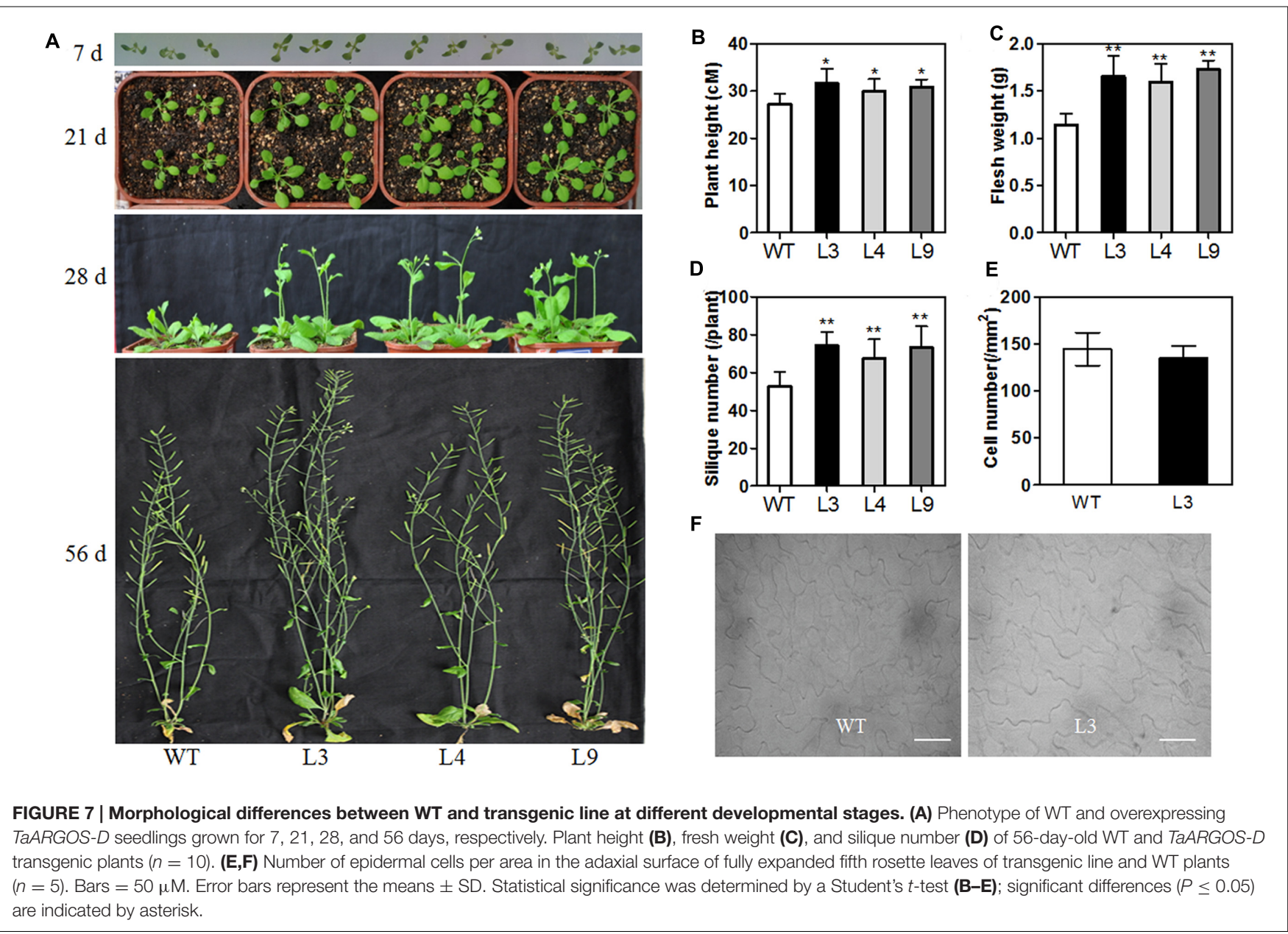




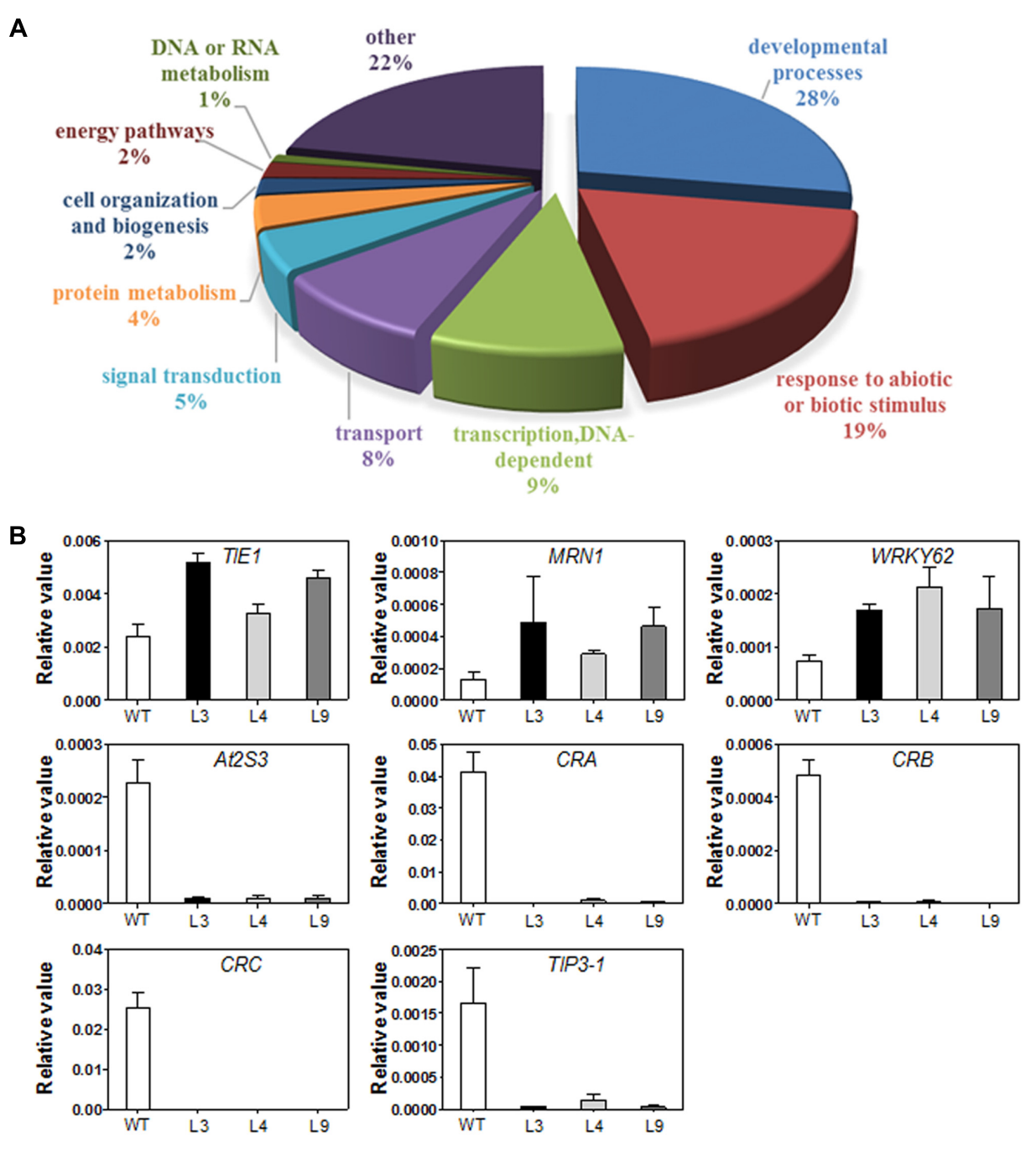

FIGURE 8 | (A) Gene ontology classification for differentially expressed genes (DEGs) in WT and TaARGOS-D transgenic plants. (B) DEGs in TaARGOS-D transgenic and WT Arabidopsis plants by qRT-PCR analysis. Error bars show SD of three replications.

of four contiguous genes, THAS1, CYP708A2, CYP705A5, and $A C T$. The second operon-like triterpene cluster (the marneral cluster) comprises the MRN1, CYP71A16, and CYP705A12 genes.

Consistent with the increased growth rate of the transgenic seedlings, several seed storage protein (SSP) genes exhibited markedly reduced expression in the transgenic seedlings compared with the WT plants (Supplementary Table S3), including three $12 \mathrm{~S}$ SSP-encoding genes (CRA1, CRB, and $C R C$ ) and four $2 S$ SSP-encoding genes (At2S1, At2S2, At2S3, and At2S5). A few ABA-related genes were also markedly inhibited in the transgenic seedlings, such as the tonoplast intrinsic proteins TIP3-1 and EM1. In addition, a number of bHLH transcription factor genes, such as bHLH38, bHLH39, bHLH1O0, and $b H L H 101$, which have been associated with irondeficiency responses and leaf morphogenesis development, were transcriptionally inhibited in the transgenic plants.

To validate the expression profiles obtained via RNA-seq, 6day-old seedlings of Col-0 and three transgenic lines (L3, L4, and
L9), grown under the same conditions used for RNA-seq, were collected for RNA extraction and qRT-PCR analysis. Eight genes selected from Supplementary Table S3 were examined, and the results were in agreement with the RNA-seq data (Figure 8B).

\section{Transgenic Arabidopsis Plants were Less Sensitive to Exogenous ABA}

The plant hormone ABA mediates plant seed germination (Jones, 2016). We showed that the abundance of several ABA-related genes was altered in our RNA-seq data (Supplementary Table S3) and that the expression of the TaARGOSs was significantly induced by $\mathrm{ABA}$ (Figure 4D). To investigate whether the TaARGOSs are involved in the ABA-mediated inhibition of seed germination, we treated the homozygous transgenic lines and WT plants with exogenous ABA. As shown in Figure 9A, the germination rates of the transgenic lines and WT plants were similar on ABA-free medium after 2 days, but those 


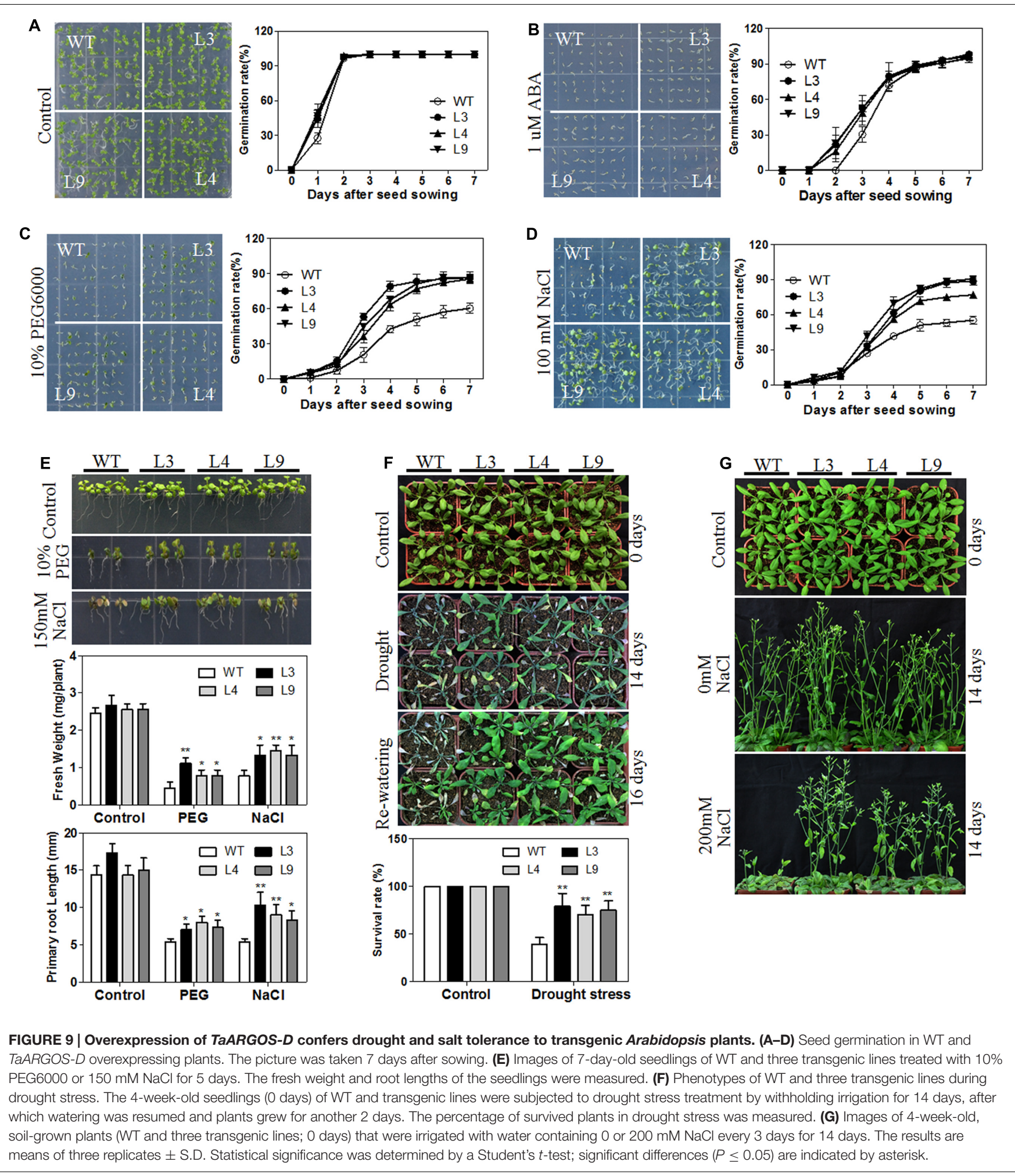

of the transgenic lines were less affected on ABA-containing medium (Figure 9B). For example, after 3 days of germination, $\sim 50 \%$ of the transgenic seeds germinated, while only $\sim 30 \%$ of the WT seeds germinated. These results demonstrated that the transgenic lines were less sensitive to ABA compared with WT plants during seed germination and that TaARGOS$D$ negatively regulates the ABA-induced inhibition of seed germination. 


\section{Overexpression of TaARGOS-D}

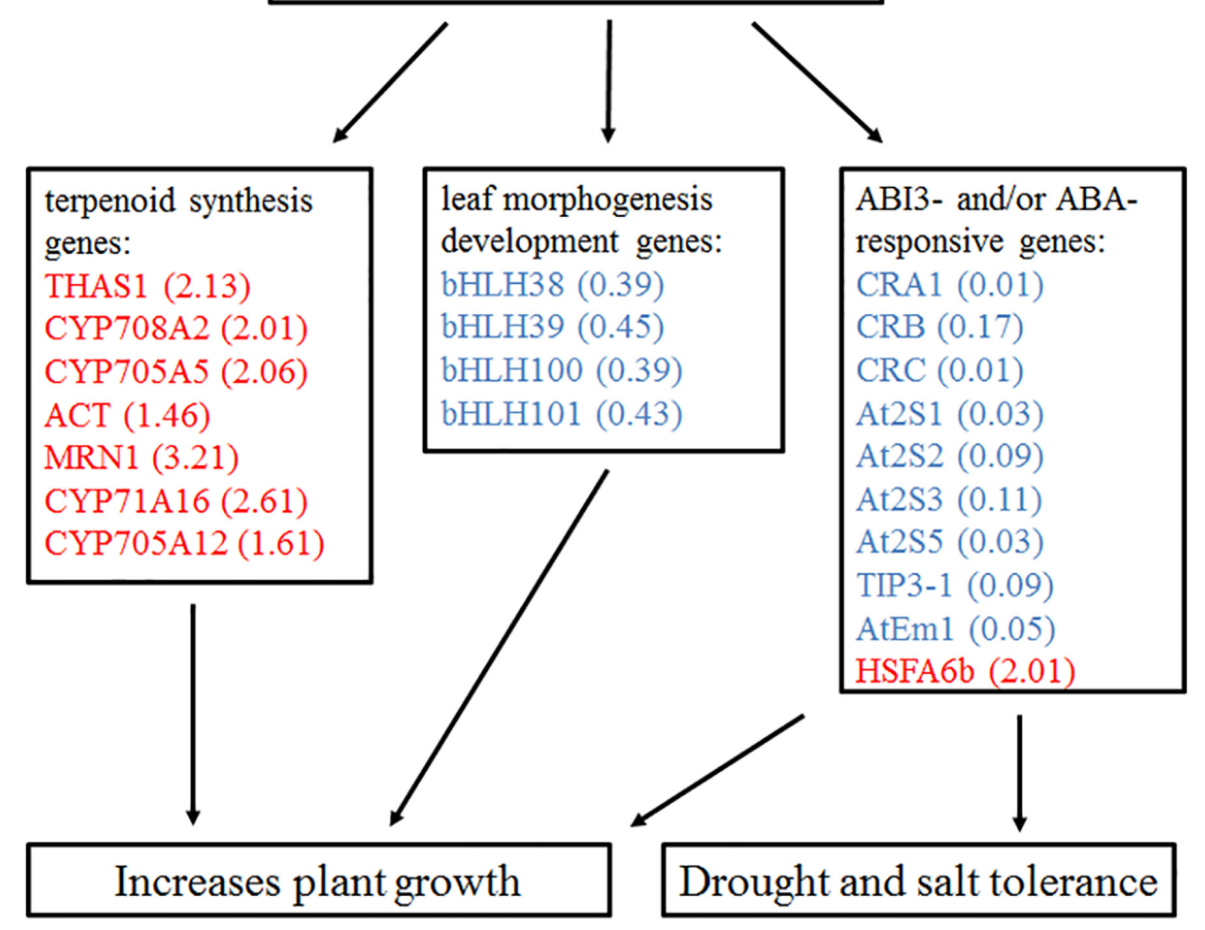

FIGURE 10 | Proposed model linking overexpression of TaARGOS-D and plant growth and stress tolerance. Red color represents up-regulated genes and blue color represents down-regulated genes. The numbers in brackets indicate the fold change of transcripts.

\section{TaARGOS-D Transgenic Plants Exhibited Improved Tolerance to Salt and Drought Stresses}

ABA has been demonstrated to be required for abiotic stress tolerance during seed germination and seedling growth (Zhu, 2002; Huang Y. et al., 2016). We next tested the germination rates of transgenic and WT plants under PEG or salt stress conditions. As shown in Figures 9C,D, the germination rates of the transgenic seeds were significantly higher than those of the WT seeds under PEG or $\mathrm{NaCl}$ treatment. Similarly sized WT and transgenic seedlings were then selected and planted vertically in either 0 or $10 \%$ PEG6000 or $150 \mathrm{mM} \mathrm{NaCl} \mathrm{MS}$ medium for 5 days. As expected, no significant differences in fresh weight and root length were observed between the transgenic and WT plants under normal conditions. However, when grown in $10 \%$ PEG6000- or $150 \mathrm{mM} \mathrm{NaCl-containing}$ MS medium, the transgenic seedlings exhibited a significantly greater fresh weight and longer roots than the WT plants, and the transgenic seedlings were less prone to chlorosis (Figure 9E).

To assess the performance of the TaARGOS-D transgenic plants under drought or salt stress in soil, the growth of the transgenic and WT plants was tested under normal or stress conditions. As expected, the transgenic plants exhibited a WT phenotype under normal conditions. However, after 2 weeks of withholding water, all of the WT plants exhibited severe wilting, and some had died, while only some of the TaARGOS$D$ transgenic plants showed signs of severe dehydration, and the rosette leaves of some of the transgenic plants were still green and fully expanded (Figure 9F). After 2 days of rewatering, only $40 \%$ of the WT plants survived, whereas 80 , 70 , and $75 \%$ of the transgenic plants survived in lines L3, L4, and L9, respectively (Figure 9F). In addition, when treated with $200 \mathrm{mM} \mathrm{NaCl}$ for 14 days, the transgenic plants were able to maintain their growth, while the WT plants exhibited significantly inhibited growth or even began to die (Figure 9G). Therefore, we can conclude that the transgenic plants are more tolerant to drought and salt stresses compared with WT plants.

\section{DISCUSSION}

In this study, we isolated and characterized the TaARGOSs, which are the homologs of reported ARGOS genes in Arabidopsis, rice and maize and encode OSR family proteins. Multiple sequence alignments showed that the TaARGOS proteins contain a conserved OSR domain with an identical LPPLPPPP motif and two putative transmembrane helices (Figure 2A). It has been reported that the OSR domain is sufficient to promote organ growth (Feng et al., 2011), thereby implying that the TaARGOSs and other plant ARGOSs share similar functions. In addition, the TaARGOSs and their orthologous in diploid, tetraploid, and 
hexaploid wheat are highly conserved (Figure 3). This high degree of conservation implies that the TaARGOSs might play essential roles in growth and development and that any sequence variation has adverse effects on plant growth.

Promoter-GUS activity assays revealed that the GUS gene was mainly expressed in vascular tissue (Figure 5B). The vascular tissue runs through the stem and branches off to different parts of the panicle. The TaARGOSs may be involved in the transport of specific molecules to the panicle through the vascular conductive system. This hypothesis is consistent with the finding that the expression of the TaARGOSs was relatively high in the stem and developing spikes (Figure 4A). In addition, most hexaploid wheat genes are present as triplicate homeologs, and a large proportion of wheat homeologs exhibit expression partitioning (Liu et al., 2015). The triplicate homeologs have three possible evolutionary fates: retention of the original function, gene silencing, and functional diversification (Shitsukawa et al., 2007). For example, Shitsukawa et al. (2007) showed that the three homoeologs genes of wheat LEAFY HULL STERILE1 (WLHS1) present genetic and epigenetic differences, and analyses of expression patterns and protein functions showed that only WLHS1-D is functional in hexaploid wheat. In the present study, different expression patterns were observed for the TaARGOSs; TaARGOS$D$ showed the highest expression level in all of the tested tissues, while TaARGOS-B was only highly expressed in the wheat stems and YS (Figure 4A). Generally, tissue-specific gene expression is associated with specific physiological and developmental functions (Zhang et al., 2012). Based on this, we speculated that TaARGOS-B may play a role in the development of stems and YS. In terms of TaARGOS-A, it might have similar functions with TaARGOS-D since they share similar tissue specific expression patterns and responses to hormones and stress treatments. These results indicated that TaARGOSs might have undergone functional diversification, the roles of TaARGOS homoeologous genes in wheat need to be further elucidated.

Similar to other identified OSR members, TaARGOS-D was localized to the ER (Figure 6). Triterpene genes thalianol synthase (THAS), marneral synthase (MRN1), and marneral oxidase (MRO) were previously shown to be ER-localized (Field et al., 2011). Interestingly, the expression levels of these genes were enhanced in the TaARGOS-D transgenic plants as revealed by RNA-seq analysis. Previous studies have shown that triterpenes protect plants against diseases and environmental stresses and are essential precursors for cell membranes and steroid hormones during plant growth and development (Field et al., 2011). Therefore, TaARGOS-D might function together with these molecules to affect the transgenic plants growth and development. In addition, the RNA-seq data also showed that in transgenic plants, four bHLH transcription factor genes (bHLH38, bHLH39, bHLH100, and bHLH101) and many seed-specific genes, including At2S3, CRC, AtEm1, and TIP3-1, were transcriptionally inhibited. Previous studies showed that the transcripts of the four $b H L H$ genes were strongly up-regulated during early leaf development and were significantly decreased afterward (Wang et al., 2007;
Andriankaja et al., 2012, 2014). The expression of seed-specific genes is gradually decreased during seedling growth (Herman and Larkins, 1999). Therefore, the decreased expression of bHLH and seed-specific genes in TaARGOS-D transgenic plants compared with that in WT indicated that the WT might be at an earlier development stage than the transgenic plants. In other words, the transgenic seedlings grew faster than the WT.

The phytohormone ABA plays an important role in plant developmental processes such as seed germination, seedling growth, and stress tolerance (Leung and Giraudat, 1998; Rock, 2000; Verma et al., 2016). ABI3, which is a central regulator in ABA signaling, represses germination and seedling development (Parcy et al., 1994). It was revealed that abi3 mutant markedly impaired the accumulation of genes At2S3, CRC, AtEm1, and TIP3-1, and the germinating seeds exhibited reduced sensitivity to exogenous ABA (Ooms et al., 1993; Parcy et al., 1994; Nakashima et al., 2006). In this study, the expression levels of the TaARGOSs were found to be increased in response to ABA treatment (Figure 4D) and overexpression of the TaARGOS-D genes decreased ABA sensitivity in seed germination (Figure 9B). Interestingly, At2S3, CRC, AtEm1, and TIP3-1, which were identified as the target genes of ABI3 (Parcy et al., 1994; Nakashima et al., 2006; Mao and Sun, 2015), were shown to be inhibited in TaARGOS-D transgenic seedlings. Taken together, we speculate that the phenotypes conferred by TaARGOS-D might be due to TaARGOS-D-mediated decreases in the expression of $A B I 3$ at the germination stage. In order to verify this hypothesis, we examined the expression of $A B I 3$ and its directly regulated genes (At2S3, CRC, and TIP3-1) at 2 days after the imbibition in overexpression lines and WT, and marked decrease of these genes were observed in overexpression lines (Supplementary Figure S4). A recent study demonstrated that HSFA6b played a pivotal role in $\mathrm{ABA}$-mediated salt and drought resistance and that overexpression of HSFA6b enhanced drought, salt, and heat stress tolerance in transgenic Arabidopsis compared with control plants (Huang Y.C. et al., 2016). Intriguingly, the RNA-seq data also revealed that HSFA6b gene expression was up-regulated in TaARGOS-D transgenic plants. Taken together, our results suggest that overexpression of TaARGOS$D$ altered the expression levels of genes involved in the terpenoid synthesis and leaf morphogenesis development, and ABI3- and/or ABA-responsive genes, thereby enhancing plant growth and conferring salt and drought tolerance in Arabidopsis (Figure 10). Future genetic and molecular biological evidence is required.

\section{AUTHOR CONTRIBUTIONS}

YZ and XT contributed equally to this work. They carried out the experiments, analyzed the results, and wrote the manuscript. MX and HP designed the experiments. QS, ZN, YY, and ZH provided the idea, instructed the research work and revised the manuscript. YL, LZ, PG, XK, and XW provided assistance to perform experiments and collect data. All authors have read and approved the final manuscript. 


\section{FUNDING}

This work was supported by the National Natural Science Foundation of China (31571747) and the National Transgenic Research Project (2016ZX08002-002).

\section{REFERENCES}

Andriankaja, M., Dhondt, S., De Bodt, S., Vanhaeren, H., Coppens, F., De Milde, L., et al. (2012). Exit from proliferation during leaf development in Arabidopsis thaliana: a not-so-gradual process. Dev. Cell 22, 64-78. doi: 10.1016/j.devcel. 2011.11.011

Andriankaja, M. E., Danisman, S., Mignolet-Spruyt, L. F., Claeys, H., Kochanke, I., Vermeersch, M., et al. (2014). Transcriptional coordination between leaf cell differentiation and chloroplast development established by TCP20 and the subgroup Ib bHLH transcription factors. Plant Mol. Biol. 85, 233-245. doi: 10.1007/s11103-014-0180-2

Clough, S. J., and Bent, A. F. (1998). Floral dip: a simplified method for Agrobacterium-mediated transformation of Arabidopsis thaliana. Plant J. 16, 735-743. doi: 10.1046/j.1365-313x.1998.00343.x

Edgerton, M. D. (2009). Increasing crop productivity to meet global needs for feed, food, and fuel. Plant Physiol. 149, 7-13. doi: 10.1104/pp.108. 130195

Fabre, D., Adriani, D. E., Dingkuhn, M., Ishimaru, T., Punzalan, B., Lafarge, T., et al. (2016). The qTSN4 effect on flag leaf size, photosynthesis and panicle size, benefits to plant grain production in rice, depending on light availability. Front. Plant Sci. 7:623. doi: 10.3389/fpls.2016. 00623

Feng, G., Qin, Z., Yan, J., Zhang, X., and Hu, Y. (2011). Arabidopsis ORGAN SIZE RELATED1 regulates organ growth and final organ size in orchestration with ARGOS and ARL. New Phytol. 191, 635-646. doi: 10.1111/j.1469-8137.2011. 03710.x

Field, B., Fiston-Lavier, A. S., Kemen, A., Geisler, K., Quesneville, H., and Osbourn, A. E. (2011). Formation of plant metabolic gene clusters within dynamic chromosomal regions. Proc. Natl. Acad. Sci. U.S.A. 108, 16116-16121. doi: $10.1073 /$ pnas. 1109273108

Field, B., and Osbourn, A. E. (2008). Metabolic diversification-independent assembly of operon-like gene clusters in different plants. Science 320, 543-547. doi: 10.1126/science. 1154990

Go, Y. S., Lee, S. B., Kim, H. J., Kim, J., Park, H. Y., Kim, J. K., et al. (2012). Identification of marneral synthase, which is critical for growth and development in Arabidopsis. Plant J. 72, 791-804. doi: 10.1111/j.1365-313X. 2012.05120.x

Gonzalez, N., Beemster, G. T., and Inzé, D. (2009). David and Goliath: what can the tiny weed Arabidopsis teach us to improve biomass production in crops? Curr. Opin. Plant Biol. 12, 157-164. doi: 10.1016/j.pbi.2008. 11.003

Guo, M., Rupe, M. A., Wei, J., Winkler, C., Goncalves-Butruille, M., Weers, B. P., et al. (2014). Maize ARGOS1 (ZAR1) transgenic alleles increase hybrid maize yield. J. Exp. Bot. 65, 249-260. doi: 10.1093/jxb/ert370

Herman, E. M., and Larkins, B. A. (1999). Protein storage bodies and vacuoles. Plant Cell 11, 601-614. doi: 10.1105/tpc.11.4.601

Hu, Y., Poh, H. M., and Chua, N. H. (2006). The Arabidopsis ARGOS-LIKE gene regulates cell expansion during organ growth. Plant J. 47, 1-9. doi: 10.1111/j. 1365-313X.2006.02750.x

Hu, Y., Xie, Q., and Chua, N. H. (2003). The Arabidopsis auxin-inducible gene ARGOS controls lateral organ size. Plant Cell 15, 1951-1961. doi: 10.1105/tpc. 013557

Hu, Z., Han, Z., Song, N., Chai, L., Yao, Y., Peng, H., et al. (2013). Epigenetic modification contributes to the expression divergence of three TaEXPA1 homoeologs in hexaploid wheat (Triticum aestivum). New Phytol. 197, 1344-1352. doi: 10.1111/nph.12131

Huang, Y., Feng, C. Z., Ye, Q., Wu, W. H., and Chen, Y. F. (2016). Arabidopsis WRKY6 transcription factor acts as a positive regulator of abscisic acid signaling during seed germination and early seedling development. PLoS Genet. 12:e1005833. doi: 10.1371/journal.pgen.1005833

\section{SUPPLEMENTARY MATERIAL}

The Supplementary Material for this article can be found online at: http://journal.frontiersin.org/article/10.3389/fpls.2017.00170/ full\#supplementary-material

Huang, Y. C., Niu, C. Y., Yang, C. R., and Jinn, T. L. (2016). The heat stress factor HSFA6b connects ABA signaling and ABA-mediated heat responses. Plant Physiol. 172, 1182-1199. doi: 10.1104/pp.16. 00860

Jefferson, R. A., Kavanagh, T. A., and Bevan, M. W. (1987). GUS fusions: betaglucuronidase as a sensitive and versatile gene fusion marker in higher plants. ЕМВО J. 6, 3901-3907.

Jones, A. M. (2016). A new look at stress: abscisic acid patterns and dynamics at high-resolution. New Phytol. 210, 38-44. doi: 10.1111/nph.13552

Langmead, B., and Salzberg, S. L. (2012). Fast gapped-read alignment with Bowtie 2. Nat. Methods 9, 357-359. doi: 10.1038/nmeth.1923

Leung, J., and Giraudat, J. (1998). Abscisic acid signal transduction. Annu. Rev. Plant Physiol. Plant Mol. Biol. 49, 199-222. doi: 10.1146/annurev.arplant.49. 1.199

Liu, Z., Xin, M., Qin, J., Peng, H., Ni, Z., Yao, Y., et al. (2015). Temporal transcriptome profiling reveals expression partitioning of homeologous genes contributing to heat and drought acclimation in wheat (Triticum aestivum L.). BMC Plant Biol. 15:152. doi: 10.1186/s12870-0150511-8

Livak, K. J., and Schmittgen, T. D. (2001). Analysis of relative gene expression data using real-time quantitative PCR and the 2(-Delta Delta C(T)) Method. Methods 25, 402-408. doi: 10.1006/meth.2001.1262

Mao, Z., and Sun, W. (2015). Arabidopsis seed-specific vacuolar aquaporins are involved in maintaining seed longevity under the control of ABSCISIC ACID INSENSITIVE 3. J. Exp. Bot. 66, 4781-4794. doi: 10.1093/jxb/ erv244

Nakashima, K., Fujita, Y., Katsura, K., Maruyama, K., Narusaka, Y., Seki, M., et al. (2006). Transcriptional regulation of ABI3- and ABA-responsive genes including RD29B and RD29A in seeds, germinating embryos, and seedlings of Arabidopsis. Plant Mol. Biol. 60, 51-68. doi: 10.1007/s11103-005-2418-5

Ooms, J., Leon-Kloosterziel, K. M., Bartels, D., Koornneef, M., and Karssen, C. M. (1993). Acquisition of desiccation tolerance and longevity in seeds of Arabidopsis thaliana (A comparative study using abscisic acidinsensitive abi3 mutants). Plant Physiol. 102, 1185-1191. doi: 10.1104/pp.102.4. 1185

Parcy, F., Valon, C., Raynal, M., Gaubier-Comella, P., Delseny, M., and Giraudat, J. (1994). Regulation of gene expression programs during Arabidopsis seed development: roles of the ABI3 locus and of endogenous abscisic acid. Plant Cell 6, 1567-1582. doi: 10.1105/tpc.6.11.1567

Qin, Z., Zhang, X., Zhang, X., Feng, G., and Hu, Y. (2014). The Arabidopsis ORGAN SIZE RELATED 2 is involved in regulation of cell expansion during organ growth. BMC Plant Biol. 14:349. doi: 10.1186/s12870-0140349-5

Quarrie, S., Pekic, Q. S., Radosevic, R., Rancic, D., Kaminska, A., Barnes, J. D., et al. (2006). Dissecting a wheat QTL for yield present in a range of environments: from the QTL to candidate genes. J. Exp. Bot. 57, 2627-2637. doi: 10.1093/jxb/ erl026

Rai, M. I., Wang, X., Thibault, D. M., Kim, H. J., Bombyk, M. M., Binder, B. M., et al. (2015). The ARGOS gene family functions in a negative feedback loop to desensitize plants to ethylene. BMC Plant Biol. 15:175. doi: 10.1186/s12870015-0554-x

Rock, C. D. (2000). Pathways to abscisic acid-regulated gene expression. New Phytol. 148, 357-396. doi: 10.1046/j.1469-8137.2000.00769.x

Rosegrant, M. W., and Cline, S. A. (2003). Global food security: challenges and policies. Science 302, 1917-1919. doi: 10.1126/science. 1092958

Shan, Q., Wang, Y., Li, J., and Gao, C. (2014). Genome editing in rice and wheat using the CRISPR/Cas system. Nat. Protoc. 9, 2395-2410. doi: 10.1038/nprot. 2014.157

Shi, J., Drummond, B., Wang, H., Archibald, R. L., and Habben, J. E. (2016a). Maize and Arabidopsis ARGOS proteins interact with ethylene receptor 
signaling complex, supporting a regulatory role for ARGOS in ethylene signal transduction. Plant Physiol. 171, 2783-2797. doi: 10.1104/pp.16.00347

Shi, J., Gao, H., Wang, H., Lafitte, H. R., Archibald, R. L., Yang, M., et al. (2016b). ARGOS8 variants generated by CRISPR-Cas9 improve maize grain yield under field drought stress conditions. Plant Biotechnol. J. 15, 207-216. doi: 10.1111/ pbi. 12603

Shi, J., Habben, J. E., Archibald, R. L., Drummond, B. J., Chamberlin, M. A., Williams, R. W., et al. (2015). Overexpression of ARGOS genes modifies plant sensitivity to ethylene, leading to improved drought tolerance in both Arabidopsis and maize. Plant Physiol. 169, 266-282. doi: 10.1104/pp.15.00780

Shitsukawa, N., Tahira, C., Kassai, K., Hirabayashi, C., Shimizu, T., Takumi, S., et al. (2007). Genetic and epigenetic alteration among three homoeologous genes of a class E MADS box gene in hexaploid wheat. Plant Cell 19, 1723-1737. doi: 10.1105/tpc.107.051813

Tamura, K., Stecher, G., Peterson, D., Filipski, A., and Kumar, S. (2013). MEGA6: molecular evolutionary genetics analysis version 6.0. Mol. Biol. Evol. 30, 2725-2729. doi: 10.1093/molbev/mst197

Tao, Q., Guo, D., Wei, B., Zhang, F., Pang, C., Jiang, H., et al. (2013). The TIE1 transcriptional repressor links TCP transcription factors with TOPLESS/TOPLESS-RELATED corepressors and modulates leaf development in Arabidopsis. Plant Cell 25, 421-437. doi: 10.1105/tpc.113.109223

Verma, V., Ravindran, P., and Kumar, P. P. (2016). Plant hormone-mediated regulation of stress responses. BMC Plant Biol. 16:86. doi: 10.1186/s12870-0160771-y

Wang, B., Sang, Y., Song, J., Gao, X., and Zhang, X. (2009). Expression of a rice OsARGOS gene in Arabidopsis promotes cell division and expansion and increases organ size. J. Genet. Genomics 36, 31-40. doi: 10.1016/S1673-8527(09) 60004-7

Wang, H. Y., Klatte, M., Jakoby, M., Baumlein, H., Weisshaar, B., and Bauer, P. (2007). Iron deficiency-mediated stress regulation of four subgroup Ib BHLH genes in Arabidopsis thaliana. Planta 226, 897-908. doi: 10.1007/s00425-0070535-x

Yoo, S. D., Cho, Y. H., and Sheen, J. (2007). Arabidopsis mesophyll protoplasts: a versatile cell system for transient gene expression analysis. Nat. Protoc. 2, 1565-1572. doi: 10.1038/nprot.2007.199

Zhang, L., Zhao, G., Jia, J., Liu, X., and Kong, X. (2012). Molecular characterization of 60 isolated wheat MYB genes and analysis of their expression during abiotic stress. J. Exp. Bot. 63, 203-214. doi: 10.1093/jxb/err264

$\mathrm{Zhu}$, J. K. (2002). Salt and drought stress signal transduction in plants. Annu. Rev. Plant Biol. 53, 247-273. doi: 10.1146/annurev.arplant.53.091401.143329

Conflict of Interest Statement: The authors declare that the research was conducted in the absence of any commercial or financial relationships that could be construed as a potential conflict of interest.

Copyright $\odot 2017$ Zhao, Tian, Li, Zhang, Guan, Kou, Wang, Xin, Hu, Yao, Ni, Sun and Peng. This is an open-access article distributed under the terms of the Creative Commons Attribution License (CC BY). The use, distribution or reproduction in other forums is permitted, provided the original author(s) or licensor are credited and that the original publication in this journal is cited, in accordance with accepted academic practice. No use, distribution or reproduction is permitted which does not comply with these terms. 